\title{
TWO FORMS OF HUMAN AMNESIA: AN ANALYSIS OF FORGETTING ${ }^{1}$
}

\author{
LARRY R. SQUIRE ${ }^{2}$ \\ Veterans Administration Medical Center, San Diego, California 92161 and Department of Psychiatry, University of California, \\ San Diego, San Diego, California 92093
}

\begin{abstract}
An analysis of forgetting was applied to patients with Korsakoff's syndrome, patients receiving bilateral electroconvulsive therapy (ECT), and case N. A. who has chronic amnesia for verbal material. Patients with Korsakoff's syndrome and case N. A. exhibited a normal forgetting rate, whereas patients receiving ECT exhibited abnormally rapid forgetting. Based on previous findings for the patient $\mathrm{H}$. M., on indirect evidence that the amnesia associated with ECI might be related to temporal lobe dysfunction, and on the diencephalic distribution of lesions in case N. A. and in Korsakoff's syndrome, these results support the hypothesis that these amnesias are distinct syndromes of memory dysfunction. Thus, the stage of memory function disrupted may be different in the two circumstances, and medial temporal and diencephalic brain regions may normally contribute in different ways to the formation of memory.
\end{abstract}

It has been known for a long time that amnesia can occur after damage in two different parts of the brain, the diencephalon and the medial temporal region. The diencephalon has been implicated in amnesia primarily as a result of neuropathological study of the alcoholic Korsakoff's syndrome. This body of work has suggested that the amnesia is associated most closely with bilateral damage to the dorsomedial thalamic nucleus and the mammillary bodies (Victor et al., 1971; Brion and Mikol, 1978; Mair et al., 1979; Brierley, 1977). In addition, radiographic evidence has shown that case $\mathrm{N}$. A., who has chronic amnesia for verbal material as the result of a penetrating brain injury, has damage in the region of the left dorsomedial thalamic nucleus (Squire and Moore, 1979). The medial temporal region was linked with memory dysfunction through study of the well known case of H. M. and of other patients who had sustained bilateral or unilateral resection of the medial temporal region for relief of intractable epilepsy (Scoville and Milner, 1957; Penfield and Milner, 1958; Milner, 1972). These resections included hippocampal formation, amygdala, uncus, and hippocampal gyrus, and the extent of memory dysfunction correlated with the extent of hippocampal removal (Milner, 1973).

\footnotetext{
${ }^{1}$ This work was supported by the Medical Research Service of the Veterans Administration and by Grant MH24600 from the National Institute of Mental Health. I thank Pamela Slater, Mary Fox. Anne Reilly, Drs. Douglas Wetzel, Neal Cohen, and Stuart Zola-Morgan for research assistance and critical discussions.

${ }^{2}$ Research career scientist at the Veterans Administration Medical Center and associate professor of psychiatry at the University of California, San Diego. To whom correspondence should be addressed at the Veterans Administration Medical Center, 3350 La Jolla Village Drive, San Diego, CA 92161.
}

In the time since Papez attributed functional significance to a circuit that included the hippocampal formation, fornix, and mammillary bodies (Papez, 1937), a common view of memory and amnesia has been that it is a unitary disorder that occurs whenever this critical pathway is damaged. Importantly, this view neither assumes nor requires that all amnesic disorders should express themselves in behavior in the same way. Thus, many of the differences among types of amnesia (Squire and Cohen, 1981; Zangwill, 1977) would be consistent with this view, if one supposed that such differences reflect cognitive deficits superimposed on a common "core" amnesia.

Recently, Huppert and Piercy have suggested that diencephalic and bitemporal amnesia might be fundamentally different entities. Using a method designed to minimize the difficulty of comparing forgetting rates between normal and amnesic groups that already differ in level of initial learning, they found that seven patients with Korsakoff's syndrome exhibited a normal rate of forgetting (Huppert and Picrcy, 1978), whereas case $\mathrm{H}$. M. exhibited an abnormal rate of forgetting (Huppert and Piercy, 1979). These data are difficult to understand as a common underlying amnesia overlaid with different degrees of other cognitive impairment. Patients with Korsakoff's syndrome, who exhibited normal forgetting, are known to have a variety of cognitive deficits other than amnesia (Butters and Cermak, 1980; Talland, 1965), whereas H. M.'s amnesia appears to be strikingly circumscribed. Indeed, Korsakoff's syndrome often has been considered to include special features not observed in bitemporal amnesia (Zangwill, 1977). Accordingly, these results raised the possibility that the underlying amnesia might be qualitatively different depending on the brain 
region affected. At the same time, this conclusion is tempered by the fact that (1) rapid forgetting was demonstrated for only a single patient and (2) the patient exhibiting rapid forgetting was considerably more amnesic than the patients with Korsakoff's syndrome.

Here, the analysis of forgetting is extended to psychiatric patients prescribed bilateral electroconvulsive therapy (ECT), case N. A., and a recently constituted population of patients with Korsakoff's syndrome identified in San Diego County. The comparison of forgetting rates was simplified by the fact that the severity of amnesia exhibited by these patients was similar (Cohen and Squire, 1981).

\section{Materials and Methods}

ECT patients. The present group consisted of eight females and three males (mean age $=39$ years; mean education $=13.2$ years) prescribed bilateral ECT for relief of depressive illness. Four patients had received a previous course of treatment, and none had received ECT within the previous 5 years. Testing was always scheduled $2 \mathrm{hr}$ after treatment at a time when anterograde amnesia is present as a relatively circumscribed disorder, in the absence of gross confusion, attentional disorders, or impairment in general intellectual capacity (Squire, 1981). For example, by $1 \mathrm{hr}$ after the fifth treatment, patients score normally on standard tests of adult intelligence.

Case N.A. This individual has been severely amnesic for verbal material since 1960 when, at the age of 22 years, he sustained a stab wound to the brain with a miniature fencing foil (Teuber et al., 1968; Squire and Slater, 1978). Recent CAT scans have identified a lesion in the left dorsal thalamus in a position corresponding to the dorsomedial nucleus (Squire and Moore, 1979). Although it is possible that other nearby structures also may have been affected by the injury, a consideration of the probable trajectory of the foil provides a reasonable basis for attributing N. A.'s amnesia to diencephalic injury, and no basis for supposing that medial temporal structures are involved (Squire and Moore, 1979). N. A. has scored above average on the Wechsler Adult Intelligence Scale (WAIS; 124 (Squire and Slater, 1978) and 124 in 1981), and aside from amnesia for verbal material and mild paresis of upward gaze, he has no other neurological impairment.

Korsakoff patients. Seven alcoholic patients with Korsakoff's syndrome residing in San Diego County participated in these studies. This group of four men and three women averaged 49 years of age, with an average of 12.9 years of education. They were identified by their normal full scale IQ $($ mean $=101$, range $=91$ to 111$)$, by their abnormal Wechsler Memory Scale score (mean $=74$, range $=64$ to 80 ), and by the presence of marked anterograde amnesia (Cohen and Squire, 1981). Independent neurological evaluation confirmed that memory impairment was the only remarkable defect of higher cortical function. All of the patients could draw a cube and a house in perspective, and none had aphasia or apraxia.

Control groups for case N. A. and Korsakoff patients. Six male Veterans Administration Hospital employees served as control subjects for N. A. They were matched to him for age (mean $=42.7$ years versus 42 years for $\mathrm{N}$. A.), education (mean $=13.9$ years versus 13 years for $N$. A.), and WAIS subtest scores (general information: $\bar{x}=$ 22.8 versus 22 for $\mathrm{N}$. A.; vocabulary: $\bar{x}=63$ versus 66 for N. A.). Eight male alcoholics, enrolled in an alcohol treatment program and free of alcohol for a minimum of 2 weeks ( $\bar{x}=17$ weeks), served as controls for the patients with Korsakoff's syndrome. As determined by structured interview, these men had an average drinking history of 22 years with no history of head injury or liver disease. They were matched to patients with Korsakoff's syndrome with respect to age (alcoholics $=55$ years, Korsakoff patients $=49$ years), education (alcoholics $=10.8$ years, Korsakoff patients $=12.9$ years), and WAIS subtest scores (information: alcoholics $=16$, Korsakoff patients $=14.9$; vocabulary: alcoholics $=44$, Korsakoff patients $=47.7$ ). The alcoholics did not exhibit anterograde amnesia, performing well above all of the amnesic patients on tests of delayed recall and paired associate learning (Cohen and Squire, 1981).

Tests: Pictures and sentences. One hundred twenty colored slides taken from magazines were used as stimulus material and presented to patients on a Kodak 400 Audioviewer. One hundred twenty additional slides served as distractor material during retention tests. The pictures test was available in four equivalent forms. One hundred twenty slides consisting of simple declarative sentences also served as stimulus material, and an additional 120 sentences served as distractor material. The sentences test was also available in four equivalent forms.

Procedure. For the learning session, patients saw 120 stimulus items (pictures or sentences) for a total of $8 \mathrm{sec}$ each. This total was achieved by presenting the entire set of items twice in succession at an exposure rate of 4 sec per item. For pictures, control subjects saw each item once for $1 \mathrm{sec}$ each. For sentences, control subjects saw each item once for $2^{1 / 2}$ sec each and, like the patients, were asked to read each sentence aloud as it was presented. Ten minutes, $2 \mathrm{hr}$, and 30 to $32 \mathrm{hr}$ after the completion of the learning session, patients and their control subjects were tested for retention by a yes-no recognition procedure. At each retention interval, subjects saw 40 of the 120 original items and 40 distractor items. Subjects within each group were assigned one of the six possible orders of three sets of retention material.

Patients with Korsakoff's syndrome and their alcoholic controls were tested on four different occasions, two for pictures and two for sentences, with at least 5 days between learning sessions. For sentences, seven of the eight alcoholic controls were tested, two of whom could be tested only once. N. A. and his control subjects were tested on two different occasions with sentences. Patients receiving ECT were tested on two different occasions for pictures $(N=9)$ or on two different occasions for sentences $(N=8)$. Six patients were tested on both pictures and sentences. One patient tested on sentences could be tested only once. Testing was scheduled on the appropriate number of occasions at $2 \mathrm{hr}$ after the third through the eighth treatments $(\bar{x}=5.0$ treatments). Patients receiving ECT were used as their own controls and tested again on two different occasions 4 months after their course of ECT, at a time when anterograde amnesia has subsided (Squire and Chace, 1975; Squire, 1981). 


\section{Results}

In all cases, retention performance was measured by calculating $d^{\prime}$, an unbiased estimate of discriminability (Green and Swets, 1966), using the percentage of hits (correct "yes" responses) and false alarms (incorrect "yes" responses). Figure 1 compares the performance of patients receiving ECT with performance of the same patients 4 months later. For both pictures and sentences, forgetting appeared to proceed more rapidly when learning occurred during the amnesia associated with ECT than when learning occurred after the amnesia had subsided. In the case of pictures (Fig. 1), where presentation time was 8 times greater during ECT than 4 months later, retention performance in the two conditions was similar at $10 \mathrm{~min}$ after learning (during ECT: $d^{\prime}=1.51$; 4 months after ECT: $d^{\prime}=1.14 ; F=3.6 ; d f=1,8 ; p>$ $0.05)$. Nevertheless, testing during the period of ECT revealed more rapid forgetting than testing 4 months after ECT, and the interaction of retention interval by testing occasion was significant $(F=8.5 ; d f=2,16 ; p<$ 0.01 ). In the case of sentences (Fig. 1), where presentation time was 3.2 times greater just after ECT than 4 months later, the results were similar. Retention performance was poorer in the amnesic condition than in the control condition $(F=36.5 ; d f=1,7 ; p<0.01)$, and forgetting was more rapid when learning occurred during the period of amnesia than when learning occurred 4 months after ECT. Because performance in the two conditions was not matched closely at the 10 -min retention interval, the interaction of retention interval by testing occasion was not significant $(F=1.1 ; d f=2,14 ; p>0.1)$. Nevertheless, the slopes of the two forgetting curves were markedly different $(F=6.0 ; d f=1,7 ; p<0.05)$.

Figure 2 compares the performance of patients with Korsakoff's syndrome and their alcoholic controls. For both pictures and sentences, the results were the same. Compared to alcoholic control subjects, patients with Korsakoff's syndrome viewed the stimulus material either 8 times longer, in the case of pictures, or 3.2 times longer, in the case of sentences. This procedure caused retention performance at $10 \mathrm{~min}$ after learning to be similar in both cases (for pictures: $F=0.04 ; d f=1,13 ; p$ $>0.1$; for sentences: $F=1.0 ; d f=1,12 ; p>0.1$ ). Over the next $32 \mathrm{hr}$, their forgetting rates were identical. The two patient groups performed similarly for both pictures and sentences (for pictures: $F=1.0 ; d f=1,13 ; p>0.1$; for sentences: $F=2.5 ; d f=1,12 ; p>0.1$ ) and there was no interaction of group by retention interval (for pictures: $F$ $=1.0 ; d f=2,26 ; p>0.1$; for sentences: $F<1.0 ; d f=2$, $24 ; p>0.1$ ).

Figure 3 compares the performance of $\mathrm{N}$. A. and his control subjects on the retention test for sentences. N. A. viewed the sentences 3.2 times longer than his controls. His performance at $10 \mathrm{~min}$ after learning, and at $2 \mathrm{hr}$ and $32 \mathrm{hr}$ after learning, fell well within the $95 \%$ confidence interval calculated for the mean of his control group.

Figure 4 compares directly the performance of ECT patients and Korsakoff patients in the pictures test. Each group viewed the pictures for a total of $8 \mathrm{sec}$. Retention was nearly identical at $10 \mathrm{~min}$ after learning (ECT patients, $d^{\prime}=1.51$; Korsakoff patients, $d^{\prime}=1.74(F=1.1 ; d f$ $=1,14 ; p>0.1)$ ). Yet, the patients receiving ECT forgot
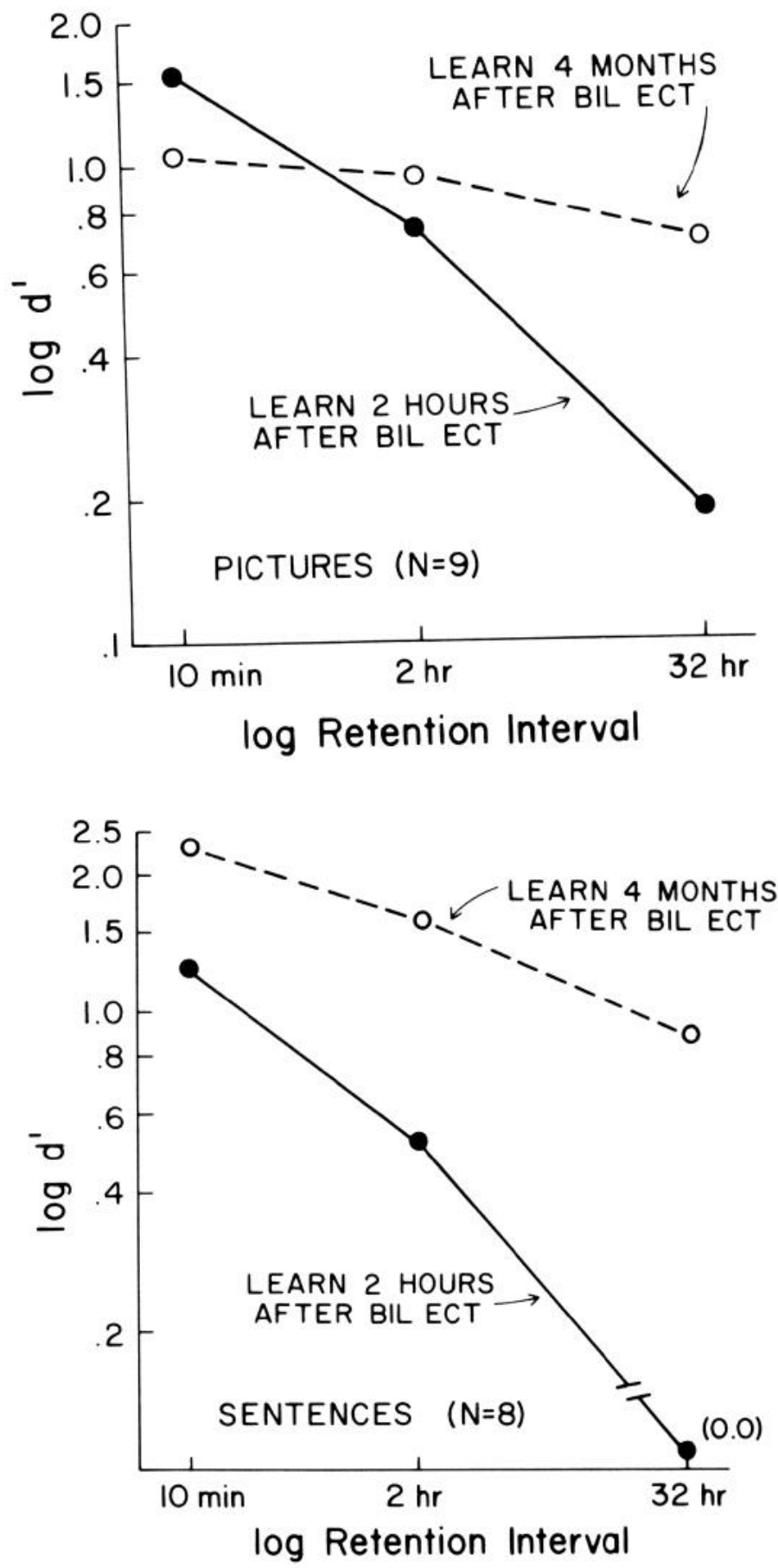

Figure 1. Retention of pictures and sentences by patients receiving ECT and by the same patients 4 months later. Two hours after treatment, patients viewed for $8 \mathrm{sec}$ each item that was to be learned. At 4 months after treatment, they viewed each picture for $1 \mathrm{sec}$ and each sentence for $2^{1 / 2}$ sec. Forgetting occurred more rapidly during the course of ECT when patients were amnesic than at 4 months after ECT when they were not amnesic. Standard errors of the mean ranged from 0.11 to 0.22 . $B I L E C T$, bilateral electroconvulsive therapy.

over the next $32 \mathrm{hr}$ at a faster rate than the patients with Korsakoff's syndrome, and the interaction of group by retention interval was significant $(F=4.0 ; d f=2,28 ; p$ $<0.05$ ). To strengthen the conclusion that patients with Korsakoff's syndrome forgot at a slower rate than pa- 

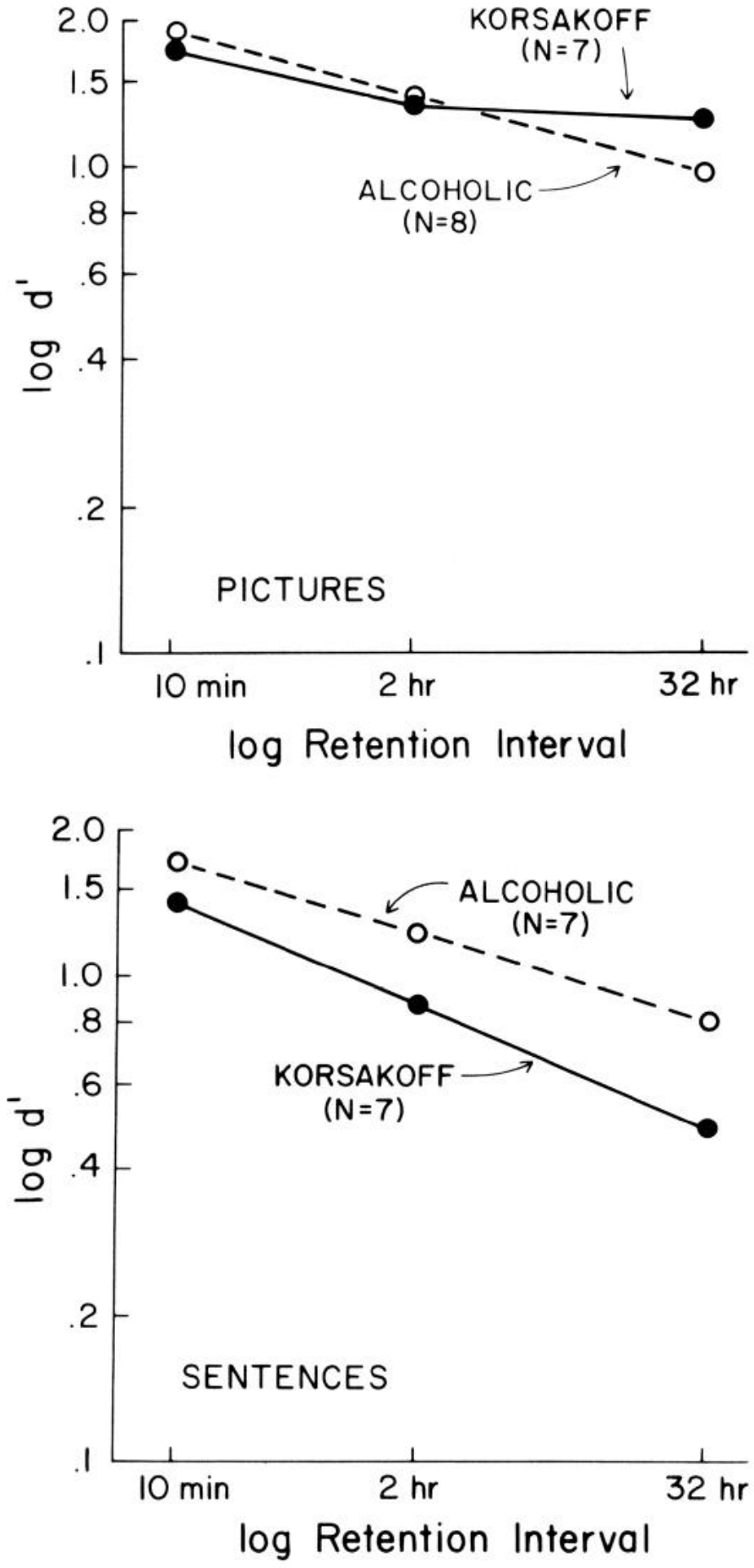

Figure 2. Retention of pictures and sentences by patients with Korsakoff's syndrome and alcoholic controls. Patients with Korsakoff's syndrome viewed for $8 \mathrm{sec}$ each item that was to be learned. Control subjects viewed each picture for $1 \mathrm{sec}$ and each sentence for $2^{1 / 2}$ sec. Forgetting occurred at the same rate for amnesic patients and control subjects. Standard errors of the mean ranged from 0.12 to 0.35 .

tients receiving ECT, a condition was established to evaluate their forgetting even when, at $10 \mathrm{~min}$ after learning, they performed more poorly than patients receiving ECT. Two new forms of the pictures test were administered to the patients with Korsakoff's syndrome,

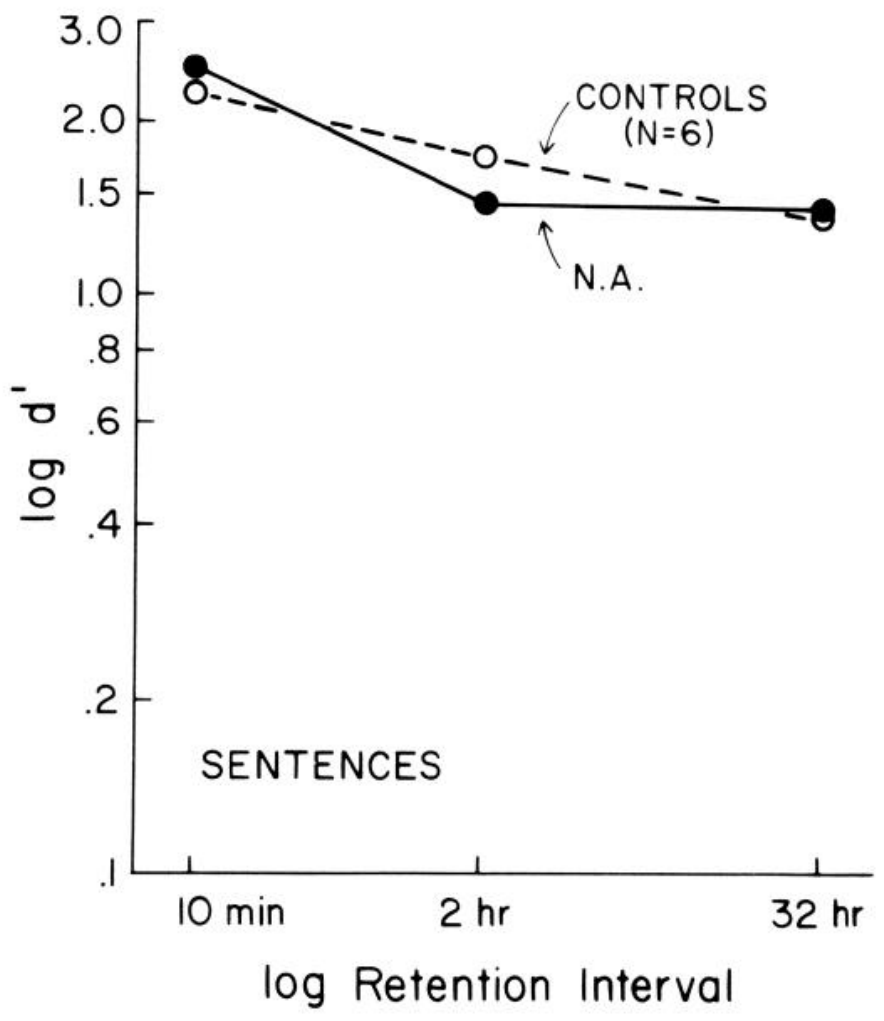

Figure 3. Retention of sentences by case N. A. and control subjects. N. A. viewed each sentence for $8 \mathrm{sec}$ and control subjects viewed each sentence for $2^{1 / 2} \mathrm{sec}$. Forgetting occurred at the same rate for N. A. as for his control subjects. For the controls, standard errors of the mean ranged from 0.21 to 0.28 .

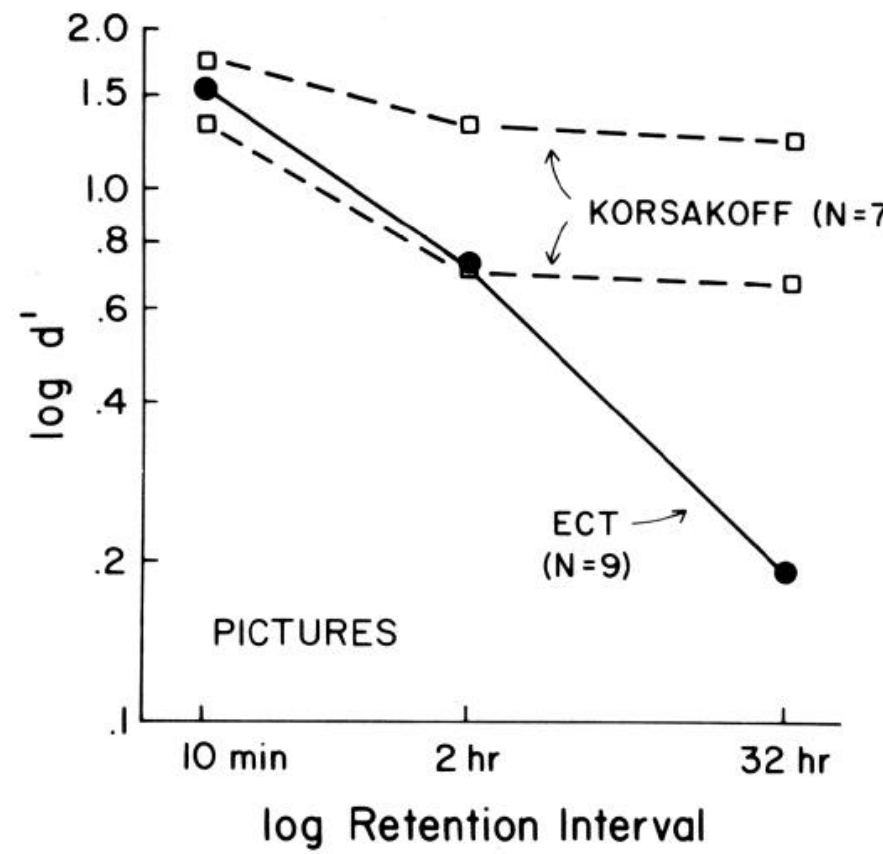

Figure 4. Retention of pictures by Korsakoff patients and by patients receiving ECT. Korsakoff patients saw each picture for either $8 \mathrm{sec}$ (upper dashed line) or $4 \mathrm{sec}$ (lower dashed line), and ECT patients saw each picture for $8 \mathrm{sec}$ (solid line). Patients receiving ECT forgot at a faster rate than patients with Korsakoff's syndrome. 
but now each item was presented only once for $4 \mathrm{sec}$, for a total exposure time of $4 \mathrm{sec}$ instead of $8 \mathrm{sec}$. Figure 4 indicates that, in this condition, retention performance at $10 \mathrm{~min}$ after learning was lower than that of ECT patients (1.31 versus 1.51 ) but that the Korsakoff patients still forgot at a more gradual rate than ECT patients. The interaction of group (Korsakoff versus ECT) by retention interval was significant $(F=3.6 ; d f=2,28 ; p$ $<0.05$ ).

\section{Discussion}

The results can be summarized by the statement that, when their retention performance at $10 \mathrm{~min}$ after learning was equated to that of control subjects, patients with Korsakoff's syndrome and case $\mathbf{N}$. A. exhibited a normal rate of forgetting. By contrast, patients receiving ECT exhibited an abnormally rapid rate of forgetting. The rapid forgetting associated with $\mathrm{ECT}$ is best attributed to ECT and not to depression or to antidepressant medication. Depression impairs immediate retention but does not cause abnormal forgetting of what is learned (Squire, 1981). Similarly, antidepressant medication does not appear to affect forgetting (Sternberg and Jarvik, 1976).

The possibility that state-dependent effects contributed to the rapid forgetting of patients receiving ECT must be raised also, since the neurological sequelae of ECT dissipate gradually during the time spanned by the learning session and the three retention tests (Fink et al., 1974). However, a role for state-dependent effects can be excluded in the present case. Possible state-dependent effects of ECT have been evaluated specifically for recognition memory of visual and verbal material. The results of these studies indicated that retention of material learned $40 \mathrm{~min}$ to $3.5 \mathrm{hr}$ after ECT was identical, regardless of whether retention was tested prior to the next ECT or at a time after the next ECT that matched the ECT-learning interval (Squire et al., 1976).

These results confirm previous findings that patients with Korsakoff's syndrome exhibit a normal forgetting rate (Huppert and Piercy, 1977) and add to the group of such patients an additional case of diencephalic amnesia, patient N. A. To case H. M., found to exhibit rapid forgetting (Huppert and Piercy, 1979), the present results add a group of patients receiving ECT. Whereas H. M.'s amnesia presumably reflects bitemporal brain damage, ECT produces a grand mal seizure; it is premature to speak with any certainty about the anatomical basis of this amnesia. Some indirect evidence, however, has linked this amnesia to dysfunction of the medial temporal region (Inglis, 1970). For example, the seizure threshold of the hippocampal formation is among the lowest of any brain region. This structure therefore might be expected to be particularly vulnerable to the effects of ECT.

The results therefore support a scheme for classifying amnesia based on behavior and on the presumed nature of the underlying neuropathology, either diencephalic or bitemporal. Recent anatomical and neuropsychological data are consistent with this proposal in the sense that the functional link between hippocampal formation, fornix, and the mammillary bodies is not so obligatory as previously thought. First, it is known now that a substantial projection from the subiculum of the hippocampal formation is directed not only through the fornix but also caudally to a variety of cortical and subcortical structures including amygdala, cingulate gyrus, entorhinal cortex, perirhinal cortex, and the medial frontal cortex (Rosene and Van Hoesen, 1977). Second, recent reviews of neurosurgical and tumor cases involving the fornix have suggested that damage to the fornix does not consistently affect memory (Squire and Moore, 1979; Squire, 1980). Fifty such cases were identified, of which only three involved memory loss. Although much of these data can be faulted for lack of neuropathological confirmation or neuropsychological testing, it is worth mentioning that the most often cited case of fornix damage and memory loss (Sweet et al., 1959) had a relatively mild amnesia (a difference between IQ and Wechsler Memory Quotient of only 13 points) compared to the amnesias described in this study and in most other contemporary studies of amnesia.

The neuropsychological separation between these amnesias suggests that the stage or aspect of memory functions impaired may be different in the two circumstances. Normal forgetting, exhibited by N. A. and by patients with Korsakoff's syndrome, supports proposals that the deficit lies in the initial encoding or registration stage of memory formation (Butters and Cermak, 1980; Huppert and Piercy, 1977; Wetzel and Squire, 1980). By this view, diencephalic amnesia is associated with a deficit in the registration of information, but the information that can be registered is maintained over time in a normal fashion. Other studies have suggested that diencephalic amnesia, at least as it is exhibited by Korsakoff patients, is also associated with a severe impairment of remote memory (Squire and Cohen, 1981; Cohen and Squire, 1981). This deficit could be a typical feature of diencephalic amnesia. However, since case N. A. appears to have good access to his remote, premorbid memory, we have suggested that impairment of remote memory implies additional lesions beyond those responsible for anterograde amnesia and may be related as well to certain cognitive deficits superimposed on amnesia (Squire and Cohen, 1981; Cohen and Squire, 1981). Further neuropathological and neuropsychological studies of diencephalic amnesia will be needed to settle this point conclusively.

Rapid forgetting, exhibited by case H. M. and by patients receiving $\mathrm{ECT}$, is consistent with proposals that the deficit lies in the consolidation or elaboration of memory during the time after initial learning (Milner, 1972; Squire, 1979). When a brain structure participating in such a process is damaged, it seems reasonable tc suppose that retention should be poorer as the interval between learning and retention increases. The status of retrograde amnesia in case $H$. M. and in patients receiv ing ECT (Marslen-Wilson and Teuber, 1975; Scoville and Milner, 1957; Cohen and Squire, 1981) is consistent with this formulation. Temporally limited retrograde amnesia, covering from 1 to 3 years, has been described clinically in case H. M. (Scoville and Milner, 1957) and has been demonstrated repeatedly by formal tests for patients receiving bilateral ECT (Cohen and Squire, 1981). Temporally limited retrograde amnesia implies the existence of a gradual process in normal memory whereby recently acquired memories become more resistant to disruption 
with the passage of time (Squire and Cohen, 1979; Squire, 1979).

Whatever the specific formulation that best accounts for normal or abnormal forgetting, the results provide additional support for the view that amnesia is not a unitary disorder. Together with previous work on this issue (Huppert and Piercy, 1977, 1979), the present results suggest that bitemporal and diencephalic brain structures, long known to be involved in memory, may contribute to normal memory functions in different ways. This proposal can be tested rigorously as additional examples of amnesia are submitted to an analysis of forgetting.

\section{References}

Brierley, J. B. (1977) Neuropathology of amnesic states. In Amnesia, C. W. M. Whitty and O. L. Zangwill, eds., pp. 199223, Butterworth \& Co., Ltd., London.

Brion, S., and J. Mikol (1978) Atteinte du noyau lateral dorsal du thalamus et syndrome de Korsakoff alcoolique. J. Neurol. Sci. 38: 249-261.

Butters, N., and L. S. Cermak (1980) Alcoholic Korsakoff's Syndrome: An Information-processing Approach to Amnesia, Academic Press, New York.

Cohen, N. J., and L. R. Squire (1981) Retrograde amnesia and remote memory impairment. Neuropsychologia, in press.

Fink, M., S. Kety, J. McGaugh, and M. Williams, eds. (1974) Psychobiology of Convulsive Therapy, V. H. Winston \& Sons, Washington, DC.

Green, D. M., and J. A. Swets (1966) A Signal Detection Theory and Psychophysics, p. 129, Robert E. Krieger, New York.

Huppert, F. A., and M. Piercy (1977) Recognition memory in amnesic patients: A defect of acquisition? Neuropsychologia 15: $643-652$.

Huppert, F. A., and M. Piercy (1978) Dissociation between learning and remembering in organic amnesia. Nature 275: $317-318$

Huppert, F. A., and M. Piercy (1979) Normal and abnormal forgetting in organic amnesia: Effect of locus of lesion. Cortex 15: 385-390.

Inglis, J. (1970) Shock, surgery and cerebral asymmetry. Br. J. Psychiatry 117: 143-148.

Mair, W. G. P., E. K. Warrington, and L. Weiskrantz (1979) Memory disorder in Korsakoff's psychosis. Brain 102: 749783.

Marslen-Wilson, W. D., and H. -L. Teuber (1975) Memory for remote events in anterograde amnesia: Recognition of public figures from news photographs. Neuropsychologia 13: 353364.

Milner, B. (1972) Disorders of learning and memory after temporal lobe lesions in man. Clin. Neurosurg. 19: 421-446.

Milner, B. (1973) Hemispheric specialization: Scope and limits. In The Neurosciences: Third Study Program, F. O. Schmitt and F. G. Worden, eds., pp. 75-89, MIT Press, Cambridge, MA.
Papez, J. W. (1937) A proposed mechanism of emotion. Arch. Neurol. Psychiatry 38: 725-743.

Penfield, W., and B. Milner (1958) Memory deficit produced by bilateral lesions in the hippocampal zone. Arch. Neurol. Psychiatry 70: 475-497.

Rosene, D. L., and G. Van Hoesen (1977) Hippocampal efferents reach widespread areas of cerebral cortex and amygdala in the rhesus monkey. Science 198: 315-317.

Scoville, W. B., and B. Milner (1957) Loss of recent memory after bilateral hippocampal lesions. J. Neurol. Neurosurg. Psychiatry 20: 11-21.

Squire, L. R. (1979) The hippocampus, space, and human amnesia. Behav. Brain Sci. 2: 514-515.

Squire, L. R. (1980) The anatomy of amnesia. Trends Neurosci. 3: $52-54$.

Squire, L. R. (1981) Neuropsychology of ECT. In Electroconvulsive Therapies: Biological Foundations and Clinical Application, W. B. Essman and R. Abrams, eds., Spectrum Publications, Jamaica, NY, in press.

Squire, L. R., and P. M. Chace (1975) Memory functions six to nine months after electroconvulsive therapy. Arch. Gen. Psychiatry 32: 1557-1564.

Squire, L. R., and N. J. Cohen (1979) Memory and amnesia: Resistance to disruption develops for years after learning. Behav. Neural. Biol. 25: 115-125.

Squire, L. R., and N. J. Cohen (1981) Remote memory, retrograde amnesia, and the neuropsychology of memory. In $\mathrm{Hu}$ man Memory and Amnesia, L. Cermak, ed., L. Erlbaum, Hillsdale, NJ, in press.

Squire, L. R., and R. Y. Moore (1979) Dorsal thalamic lesion in a noted case of chronic memory dysfunction. Ann. Neurol. 6 : 503-506.

Squire, L. R., and P. C. Slater (1978) Anterograde and retrograde memory impairment in chronic amnesia. Neuropsy. chologia 16: 313-322.

Squire, L. R., P. C. Slater, and P. M. Chace (1976) Anterograde amnesia following electroconvulsive therapy: No evidence for state-dependent learning. Behav. Biol. 17: 31-41.

Sternberg, E. D., and M. E. Jarvik (1976) Memory functions in depression. Arch. Gen. Psychiatry 33: 219-224.

Sweet, W. H., G. A. 'I'alland, and F. R. Ervin (1959) Loss of recent memory following section of fornix. Trans. Am. Neurol. Assoc. 84: 76-82.

Talland, G. A. (1965) Deranged Memory, Academic Press, New York.

Teuber, H. -L., B. Milner, and H. G. Vaughan (1968) Persistent anterograde amnesia after stab wound of the basal brain. Neuropsychologia 6: 267-282.

Victor, M., R. Adams, and G. Collins (1971) The WernickeKorsakoff Syndrome, F. A. Davis, Philadelphia.

Wetzel, C. D., and L. R. Squire (1980) Encoding in anterograde amnesia. Neuropsychologia 18: 177-184.

Zangwill, O. L. (1977) The amnesic syndrome. In Amnesia, C. W. M. Whitty and O. L. Zangwill, eds., pp. 104-117, Butterworth \& Co., Ltd., London. 